\title{
Solid-State Electrolyte-Gated Graphene in Optical Modulators
}

DOI:

10.1002/adma.201606372

\section{Document Version}

Accepted author manuscript

Link to publication record in Manchester Research Explorer

\section{Citation for published version (APA):}

Rodriguez, F. J., Aznakayeva, D. E., Marshall, O. P., Kravets, V. G., \& Grigorenko, A. N. (2017). Solid-State

Electrolyte-Gated Graphene in Optical Modulators. Advanced Materials. https://doi.org/10.1002/adma.201606372

\section{Published in:}

Advanced Materials

\section{Citing this paper}

Please note that where the full-text provided on Manchester Research Explorer is the Author Accepted Manuscript or Proof version this may differ from the final Published version. If citing, it is advised that you check and use the publisher's definitive version.

\section{General rights}

Copyright and moral rights for the publications made accessible in the Research Explorer are retained by the authors and/or other copyright owners and it is a condition of accessing publications that users recognise and abide by the legal requirements associated with these rights.

\section{Takedown policy}

If you believe that this document breaches copyright please refer to the University of Manchester's Takedown Procedures [http://man.ac.uk/04Y6Bo] or contact uml.scholarlycommunications@manchester.ac.uk providing relevant details, so we can investigate your claim.

\section{OPEN ACCESS}




\section{WILEY-VCH}

DOI: $10.1002 /(($ please add manuscript number $))$

Article type: Communication

\section{Solid-state electrolyte gated graphene in optical modulators}

Francisco J. Rodriguez*, Diana E. Aznakayeva, Owen P. Marshall, Vasyl G. Kravets, Alexander N. Grigorenko

School of Physics and Astronomy, the University of Manchester, M13 9PL, UK

* E-mail: francisco.rodriguez@manchester.ac.uk

Keywords: graphene heterostructures, Pauli blocking, $\mathrm{HfO}_{2}$, field effect transistors, double layers

The gate-tunable wide-band absorption of graphene makes it suitable for light modulation from terahertz to visible light. The realization of graphene-based modulators, however, faces challenges connected with graphene's low absorption and the high electric fields necessary to change graphene's optical conductivity. Here we demonstrate a solid state supercapacitor effect with the high-k dielectric hafnium oxide that allows modulation from the near-infrared to shorter wavelengths close to the visible spectrum with remarkably low voltages $(\sim 3 \mathrm{~V})$.

The electro-absorption modulators are based on a Fabry-Perot resonator geometry that allows modulation depths over $30 \%$ for free-space beams. 


\section{WILEY-VCH}

Graphene, with only one atom thickness, has a relatively large $2.3 \%$ absorption which is constant from the mid infrared to the visible. ${ }^{[1]}$ This absorption originates from interband transitions and can be switched on and off by a shift in the Fermi level below which Pauli blocking occurs. ${ }^{[2]}$ The shift in Fermi level can be controlled by electric field gating, making graphene particularly attractive for electro-optical modulators ${ }^{[3]}$ However, the absorption of a single layer of graphene is too weak for most applications. Fortunately, there are several ways to deal with this problem ${ }^{[4]}$ for example by using structured graphene stacks, ${ }^{[5]}$ implementing smart photonic structures, ${ }^{[6,7]}$ or using hybrid graphene-plasmonic devices. ${ }^{[8]}$ Fast optical modulators have been demonstrated where graphene was used to modulate light in a waveguide. ${ }^{[9]}$ In such cases, light interacts with graphene not in a single pass through the atom thick layer, but over a long distance in the graphene plane. In contrast, less progress has been made for optical modulators working with free-space beams in the near infrared or visible spectrum. A fast but weak modulator based on a reflective structure with a quarterwave separator has been demonstrated ${ }^{[10]}$ and applied for intracavity stabilization of a frequency comb mode-locked laser. ${ }^{[11]}$ Others have used high refractive index contrast gratings. ${ }^{[12]}$ On the other hand, numerical simulations have predicted modulation depths approaching unity with low insertion loses in resonant structures formed with multilayer dielectric mirrors. ${ }^{[13]}$

One crucial aspect of graphene-based modulators is the choice of the separator dielectric. Indeed, the electric fields necessary to produce a change in graphene's absorption at wavelengths shorter than $2 \mu \mathrm{m}$ are at the limit for dielectric breakdown of most materials. The modulation of the optical properties of graphene in the near infrared $(1.5 \mu \mathrm{m})$ requires high quality dielectrics, normally very thin layers $(\sim 10 \mathrm{~nm})$ grown by atomic layer deposition (ALD), or ionic liquids. At shorter wavelengths the challenge is even bigger, and only ionic 


\section{WILEY-VCH}

liquids have been demonstrated down to visible wavelengths. ${ }^{[14,15]}$ However, ionic liquids tend to have a slow speed of response and may not be suitable for some device structures. Here we demonstrate that hafnium oxide deposited by electron beam evaporation allows gating graphene with surprisingly low voltages from the near infrared to wavelengths approaching the visible range. Our electro-absorption modulators are based on a Fabry-Perot geometry. Multiple reflections inside the optical cavity enhance the light-graphene interaction, thereby amplifying the modulation effect. Instead of the complex multilayer Bragg reflector mirrors used for graphene-based photodetection, ${ }^{[7]}$ we have opted for thin metal mirrors on both sides which show a good optical performance at the target wavelengths and also serve to provide two separate electrical gates. The observed electrochemical effect allows gating graphene with low voltages independently of the dielectric thickness, which can be adjusted to obtain a resonance at the desired wavelength.

The typical device design (Figure 1) consists of two metal electrodes that simultaneously act as electrical gates and the optical reflectors of the Fabry-Perot cavity. A graphene active layer is embedded at the center of the cavity and electrically contacted at the edges. The dielectric (hafnium oxide) has a thickness $D$ both above and below graphene corresponding to approximately $\lambda / 4 n$, where $\lambda$ is the operation wavelength of the modulator and $n$ the refractive index. Thin layers of copper, gold or silver were used as semi-transparent electrodes in different devices. In general, these devices can work both in transmission and reflection, but they can be optimized to work only in reflection with a thicker bottom gate.

Firstly we studied a simple modulator with a graphene/dielectric/metal structure, as shown in the inset in Figure 2a. The dielectric was a $175 \mathrm{~nm}$ thick layer of hafnium oxide separating graphene from a $70 \mathrm{~nm}$ thick copper layer. The dielectric thickness corresponds to a quarter- 


\section{WILEY-VCH}

wave separation for $1.5 \mu \mathrm{m}$ wavelength. Optical spectra were acquired with a Bruker HYPERION Fourier transform infrared (FTIR) microscope. The device featured a broad resonance in reflection around $1.5 \mu \mathrm{m}$ and provided a maximum optical modulation depth of $7 \%$ with only $8 \%$ insertion losses (Figure $2 a$ ). We then compared the changes in the reflectivity at different gate voltages (Figure 2c) with the results from a Fresnel transfer matrix model of the device for different Fermi energies in graphene (Figure 2d). A good match is found for Fermi energies that would correspond to a much thinner dielectric in a conventional plane-parallel capacitor model. In our case, an effective $20 \mathrm{~nm} \mathrm{HfO}_{2}$ thickness is estimated from the modelling even though the real thickness was $175 \mathrm{~nm}$ (see Supporting Information for calculation details). We also found that the electrical resistance of graphene showed variations at gate voltages that were much smaller than expected for the experimental oxide thickness (Figure 2b). Moreover, a negative hysteresis was observed. Most dielectrics used for graphene field effect transistors (FET) tend to show a positive hysteresis, which means that mobile ions or dipoles are effectively screening the applied electric field. ${ }^{[16]}$ The negative hysteresis means that in this case the electric field that affects graphene is amplified. These results are similar to the effect of an electrical double layer.

To elucidate the properties of the hafnium oxide layer, we studied the dynamics of the gating effect. In this case, the optical measurements were performed with a white light source and a near infrared spectrometer. Figure $2 \mathrm{e}$ shows how the reflectance and the electrical resistance of graphene change with jumps of the gate voltage in a similar graphene/dielectric/metal structure with $85 \mathrm{~nm}$ thick oxide. Due to the reduced oxide thickness, the measured wavelengths are far from the resonance, which makes the modulation weaker. When we switch the gate voltage from the charge neutrality point $(1 \mathrm{~V})$ to $-1.8 \mathrm{~V}$ we observe an increase in the reflectivity that takes place in a quite slow ( 10 seconds) time scale. When the 


\section{WILEY-VCH}

gate voltage is taken back to $1 \mathrm{~V}$ the reflectivity quickly goes back to the initial value. The return was faster than the time resolution of our measurements $(<20 \mathrm{~ms})$. This behavior suggests that we have two different time scales in the response to the applied gating voltage. One is slow, corresponding to the large capacitance provided by the electrical double layerlike effect. And the other one is extremely fast, corresponding to a smaller capacitance in parallel that we presume is related to the plane parallel capacitor with dielectric thickness approximately equal to the actual thickness of the hafnium oxide layer (see Supporting Information). The striking difference in the rise and decay times can be easily understood through the dependence of the device reflectance on the Fermi energy of graphene. Small changes in the Fermi energy around the charge neutrality point will not affect the optical properties in the near infrared. However, the changes around the energy levels close to the Pauli blocking will have an effect on the absorption at the tested wavelengths. When the change in Fermi energy produced by a gate voltage change has a fast initial response, followed by a slower one, it will be the latter which affects the rise in the reflectance but the initial fast contribution which dictates the decay. In contrast, the resistance of graphene is more strongly influenced by gating when the Fermi energy is close to the charge neutrality point. The slow component of the time response is comparable to other electrolyte gated devices. ${ }^{[15,17]}$

To check the behavior of the slow response with temperature, we measured the electrical properties of graphene using a cryostat partially evacuated with a rotary pump (Figure 2f). Similar results were also obtained in a nitrogen atmosphere. For the sake of clarity, only the positive direction of the voltage sweeps $(0.083 \mathrm{~V} / \mathrm{s})$ are presented, along with the positive values of the current on a logarithmic scale. As the temperature is reduced, the charge neutrality point in the resistance curves is shifted towards increasingly positive voltages, and 


\section{WILEY-VCH}

the width of the peak is broadened. The gate current is also significantly reduced at lower temperatures. All this corresponds to an increase in the effective thickness of the separator layer, which is explained by a lower ionic mobility in the hafnium oxide.

We briefly consider a physical mechanism for the observed "supercapacitance" effect. It is known that oxides can conduct electricity in strong electric fields ${ }^{[18]}$ or due to nonstoichiometry. ${ }^{[19]}$ More specifically, it has been found that hafnium oxide also conducts in strong electric fields ${ }^{[20]}$ and its ionic mobility has been studied for memristor devices. ${ }^{[21]}$ Hence, we suggest that the application of the electric field to hafnium oxide leads to an ionic current which produces an electric double layer near the graphene surface. The exact nature of the double layer is not clear at the moment, but its thickness could be connected to the roughness of the oxide layer as the atomic force microscope (AFM) measurement in the Supporting Information reveals. The situation is complicated by the fact that light illumination could introduce photoinduced doping in atomic heterostructures ${ }^{[22]}$ and hafnium oxide could show ferroelectric characteristics ${ }^{[23]}$.

The $\sim 7 \%$ modulation depth of the quarter-wave separator structure (Figure 2c), although already significantly higher than the single pass absorption in graphene, can be improved further. To increase the modulation depth, a Fabry-Perot resonator was completed by adding another quarter-wave separator and a partially transmitting mirror on the top of the structure. In this structure, graphene is completely encapsulated, which demonstrates that the supercapacitance effect is independent of the contact with the atmosphere. Additionally, the Fabry-Perot resonator with metallic mirrors allows one to use a double gate which increases the induced Fermi energy change and hence the Pauli blocking wavelengths at which the device can be modulated efficiently. 


\section{WILEY-VCH}

The results from a device with copper gates $\left(\mathrm{d}_{1}=30 \mathrm{~nm}, \mathrm{~d}_{2}=70 \mathrm{~nm}\right)$ designed to operate near $1.55 \mu \mathrm{m}$ wavelength are shown in Figure 3a. The reflection spectra show a modulation depth of $20 \%$ between gate voltages $-1 \mathrm{~V}$ and $-3 \mathrm{~V}$. The insertion losses are $45 \%$. Numerical simulations based on Fresnel theory reproduce well the measured data for shifts of the Fermi energy of at least $0.7 \mathrm{eV}$ (Figure 3b). We would like to note that the refractive index of metals such as copper or silver is highly dependent on the deposition conditions. Simulations using the refractive index reported in the literature for optimized processes yield modulation depths of more than $90 \%$ for the same shifts of the Fermi energy (see Figure S1). On the other hand, the evaporation of hafnium oxide can produce a non-stoichiometric film with a small absorption that can strongly decrease the effect of the graphene modulation due to the effect of the cavity. Thus an optimization of the materials and processes could lead to much improved modulation depths. The results from another device operating around $1.1 \mu \mathrm{m}$ wavelength both in transmission and in reflection are shown in Figures $3 \mathrm{c}$ and $3 \mathrm{~d}$. In this case, the hafnium oxide thickness was $D=100 \mathrm{~nm}$, the top gate gold $\left(\mathrm{d}_{1}=30 \mathrm{~nm}\right)$ and the bottom gate copper $\left(\mathrm{d}_{2}=30 \mathrm{~nm}\right)$. In reflection, the insertion loses are close to $50 \%$ and the modulation depth $13 \%$. In transmission, the insertion losses grow to $90 \%$ but the modulation depth also grows to $28 \%$. Finally, properties of a device operating around $830 \mathrm{~nm}$ are represented in Figures 3e-g. The hafnium oxide thickness was $D=70 \mathrm{~nm}$ and both gates were made out of $30 \mathrm{~nm}$ thick silver. In the devices operating in transmission, the Fabry-Perot cavity acts as a band pass filter. When illuminated with white light, all the light in the transmission band is modulated. Thus, in this device, it was possible to observe the modulation in images taken with a silicon charge-coupled device (CCD) sensor using broadband illumination. 


\section{WILEY-VCH}

As described above, our devices display two distinct time scales: they reach a stable state in seconds but they also have an extremely fast initial response. We investigated the response to high frequency signals (using the device from Figure 3a) by applying a $1 \mathrm{MHz}$ sinusoidal signal to the gate and measuring the reflectance of a laser diode beam of $1571 \mathrm{~nm}$ wavelength (Figure 4a). The device reflectance followed the $1 \mathrm{MHz}$ signal, although the voltages required to achieve strong modulation depth are higher than in the slow measurements. On the other hand, no damage was observed in the device after such voltages are applied at high frequency. The modulated optical output changes from a $180^{\circ}$ phase-shifted signal at $0 \mathrm{~V}$ offset to a double frequency signal at $-0.6 \mathrm{~V}$ and to a signal with much bigger amplitude and the same phase as the excitation voltage at $-1.2 \mathrm{~V}$ offset. The modulation depth reached $40 \%$ in this latter case. The fast rise and decay in the modulation obtained with a square signal of $14 \mathrm{~V}$ peak-to-peak and $-0.9 \mathrm{~V}$ offset (Figure $4 \mathrm{~b}$ ) demonstrates that the speed of response of the device can in fact be much higher and we were only limited by our measurement system. In Figure $4 c$ we show the reflectance curve obtained with slow changes of the gate voltage. Superposed onto the experimental results, we also present the numerical simulation of the reflectance at the estimated Fermi energy. If we compare the traces in Figure 4a with the reflectance curve in Figure 4c it becomes evident why the gate voltage offset is a determining factor for the modulation. At $0 \mathrm{~V}$ offset, the modulation happens around $0.3 \mathrm{eV}$, with the negative slope of the reflectance curve giving the $180^{\circ}$ phase shift. At $-0.6 \mathrm{~V}$ offset, the modulation happens around the peak in the reflectance curve at $0.4 \mathrm{eV}$, producing the double frequency response. Finally, at $-1.2 \mathrm{~V}$ offset the modulation happens in the positive and steeper slope which yields the strongest modulation. To show that our devices can operate close to the visible region, the modulation of a white light source by the device of Figure $3 \mathrm{c}$ is shown in Figure 4d. The electrical signal applied was a $2 \mathrm{~Hz}$ sinusoidal function with $8 \mathrm{~V}$ peak-to-peak and $0 \mathrm{~V}$ offset. The modulation in this case primarily arises from the slow 


\section{WILEY-VCH}

mechanism, with the double frequency asymmetric response stemming from the alternating movement of the Fermi energy to both sides of the charge neutrality point.

The Fabry-Perot geometry with metallic mirrors and hafnium oxide separators allowed us to build efficient graphene-based electro-absorption modulators. By harnessing the supercapacitor effect found in hafnium oxide, we have created optical modulators working at small voltages and at wavelengths approaching the visible. Although the strong modulation response observed with small gate voltages is relatively slow, as it happens also with other electrolyte gated devices, it can be used to shift the Fermi level close to the desired energy range with a DC voltage. The plane parallel capacitor structure then allows the modulation at higher frequencies. In principle, the device area can be reduced down to a diffraction limited laser spot, which would reduce the high frequency capacitance and allow extremely high operation speed. With additional optimization, our devices should also be able to modulate light at visible frequencies.

\section{Experimental Section}

Device fabrication: The typical device fabrication starts with the deposition by e-beam evaporation of the bottom electrode on a quartz substrate using a shadow mask. $1 \mathrm{~nm}$ of $\mathrm{Cr}$ is deposited first for better adhesion followed by the required thickness of $\mathrm{Cu}, \mathrm{Ag}$ or $\mathrm{Au}$. A 10 $\mathrm{nm}$ layer of hafnium oxide is evaporated without breaking the vacuum to prevent degradation of $\mathrm{Cu}$ or $\mathrm{Ag}$. Next the shadow mask is replaced by a wider one and the required thickness of hafnium oxide is evaporated. CVD graphene grown on copper foil is spin coated with a layer of PMMA for support. The copper substrate is etched in a solution of ammonium persulfate and the floating membrane is cleaned in subsequent baths of deionized water. The membrane is then transferred to the device and baked at $170^{\circ} \mathrm{C}$ for 10 minutes to facilitate adhesion to the substrate. The PMMA layer is removed in acetone and the device rinsed in isopropanol. 


\section{WILEY-VCH}

Contacts to the graphene are defined by photolithography and deposited by e-beam evaporation of $\mathrm{Au}$ with a $\mathrm{Cr}$ adhesion layer. Photolithography with a negative tone resist is used to protect graphene in the active area of the device and the graphene from the rest of the surface is removed by Ar: $\mathrm{O}_{2}$ plasma etching. Another hafnium oxide layer is evaporated while protecting the external part of the contacts with a shadow mask. The top electrode is defined with photolithography and the required metal deposited again by e-beam evaporation, with $\mathrm{Cu}$ and $\mathrm{Ag}$ protected by the subsequent evaporation of $10 \mathrm{~nm}$ of hafnium oxide without breaking the vacuum. This protective layer does not affect the optical properties.

\section{Supporting Information}

Supporting Information is available from the Wiley Online Library or from the author.

\section{Acknowledgements}

The work has been supported by European Commission under Graphene Flagship (contract no. CNECT-ICT-604391). We thank Dmitro Kundys for assistance with the optical measurement setup and Artem Mishchenko for the capacitance measurements.

Received: ((will be filled in by the editorial staff))

Revised: ((will be filled in by the editorial staff))

Published online: ((will be filled in by the editorial staff)) 
References

[1] R. R. Nair, A. N. Grigorenko, P. Blake, K. S. Novoselov, T. J. Booth, N. M. R. Peres, T. Stauber, A. K. Geim, Science 2008, 320, 1308.

[2] F. Wang, Y. Zhang, C. Tian, C. Girit, A. Zettl, M. Crommie, Y. R. Shen, Science 2008, 320, 206.

[3] F. Bonaccorso, Z. Sun, T. Hasan, A. C. Ferrari, Nat. Photonics 2010, 4, 611; Z. P. Sun, A. Martinez, F. Wang, Nat. Photonics 2016, 10, 227.

[4] A. Grigorenko, M. Polini, K. Novoselov, Nat. Photonics 2012, 6, 749.

[5] H. Yan, X. Li, B. Chandra, G. Tulevski, Y. Wu, M. Freitag, W. Zhu, P. Avouris, F. Xia, Nat. Nanotechnol. 2012, 7, 330.

[6] T. Mueller, F. N. A. Xia, P. Avouris, Nat. Photonics 2010, 4, 297; Y. D. Gao, R. J. Shiue, X. T. Gan, L. Z. Li, C. Peng, I. Meric, L. Wang, A. Szep, D. Walker, J. Hone, D. Englund, Nano Lett. 2015, 15, 2001; X. T. Gan, R. J. Shiue, Y. D. Gao, K. F. Mak, X. W. Yao, L. Z. Li, A. Szep, D. Walker, J. Hone, T. F. Heinz, D. Englund, Nano Lett. 2013, 13, 691; X. T. Gan, K. F. Mak, Y. D. Gao, Y. M. You, F. Hatami, J. Hone, T. F. Heinz, D. Englund, Nano Lett. 2012, 12, 5626.

[7] M. Furchi, A. Urich, A. Pospischil, G. Lilley, K. Unterrainer, H. Detz, P. Klang, A. M. Andrews, W. Schrenk, G. Strasser, T. Mueller, Nano Lett. 2012, 12, 2773.

[8] T. J. Echtermeyer, L. Britnell, P. K. Jasnos, A. Lombardo, R. V. Gorbachev, A. N. Grigorenko, A. K. Geim, A. C. Ferrari, K. S. Novoselov, Nat. Commun. 2011, 2, 458; V. G. Kravets, F. Schedin, R. Jalil, L. Britnell, K. S. Novoselov, A. N. Grigorenko, J. Phys. Chem. C 2012, 116, 3882; B. D. Thackray, P. A. Thomas, G. H. Auton, F. J. Rodriguez, O. P. Marshall, V. G. Kravets, A. N. Grigorenko, Nano Lett. 2015, 15, 3519.

[9] M. Liu, X. Yin, E. Ulin-Avila, B. Geng, T. Zentgraf, L. Ju, F. Wang, X. Zhang, Nature 2011, 474, 64; C. T. Phare, Y.-H. Daniel Lee, J. Cardenas, M. Lipson, Nat. Photonics 


\section{WILEY-VCH}

2015, 9, 511; N. Youngblood, Y. Anugrah, R. Ma, S. J. Koester, M. Li, Nano Lett. 2014, 14, 2741; M. Mohsin, D. Schall, M. Otto, A. Noculak, D. Neumaier, H. Kurz, Opt. Express 2014, 22, 15292; D. Ansell, I. P. Radko, Z. Han, F. J. Rodriguez, S. I. Bozhevolnyi, A. N.

Grigorenko, Nat. Commun. 2015, 6, 8846.

[10] C.-C. Lee, S. Suzuki, W. Xie, T. R. Schibli, Opt. Express 2012, 20, 5264.

[11] C. C. Lee, C. Mohr, J. Bethge, S. Suzuki, M. E. Fermann, I. Hartl, T. R. Schibli, Opt. Lett. 2012, 37, 3084.

[12] T. Sun, J. Kim, J. M. Yuk, A. Zettl, F. Wang, C. Chang-hasnain, Opt. Express 2016, $24,26035$.

[13] R. W. Yu, V. Pruneri, F. J. G. de Abajo, ACS Photonics 2015, 2, 550; B. Vasić, R. Gajić, Opt. Lett. 2014, 39, 6253.

[14] E. O. Polat, C. Kocabas, Nano Lett. 2013, 13, 5851.

[15] E. O. Polat, O. Balci, C. Kocabas, Sci. Rep. 2014, 4, 6484.

[16] A. Veligura, P. J. Zomer, I. J. Vera-Marun, C. Józsa, P. I. Gordiichuk, B. J. van Wees, J. Appl. Phys. 2011, 110, 113708.

[17] J. Sayago, U. Shafique, F. Soavi, F. Cicoira, C. Santato, J. Mater. Chem. C 2014, 2 , 10273.

[18] G. Dearnaley, A. M. Stoneham, D. V. Morgan, Rep. Prog. Phys. 1970, 33, 1129.

[19] P. Kofstad, Nonstoichiometry, diffusion, and electrical conductivity in binary metal oxides, Wiley-Interscience New York, 1972.

[20] N. A. Chowdhury, R. Garg, D. Misra, Appl. Phys. Lett. 2004, 85, 3289.

[21] A. Wedig, M. Luebben, D. Y. Cho, M. Moors, K. Skaja, V. Rana, T. Hasegawa, K. K. Adepalli, B. Yildiz, R. Waser, I. Valov, Nat. Nanotechnol. 2016, 11, 67. 
[22] L. Ju, J. Velasco, Jr., E. Huang, S. Kahn, C. Nosiglia, H. Z. Tsai, W. Yang, T.

Taniguchi, K. Watanabe, Y. Zhang, G. Zhang, M. Crommie, A. Zettl, F. Wang, Nat.

Nanotechnol. 2014, 9, 348.

[23] J. Muller, P. Polakowski, S. Mueller, T. Mikolajick, Ecs J Solid State Sc 2015, 4, N30.
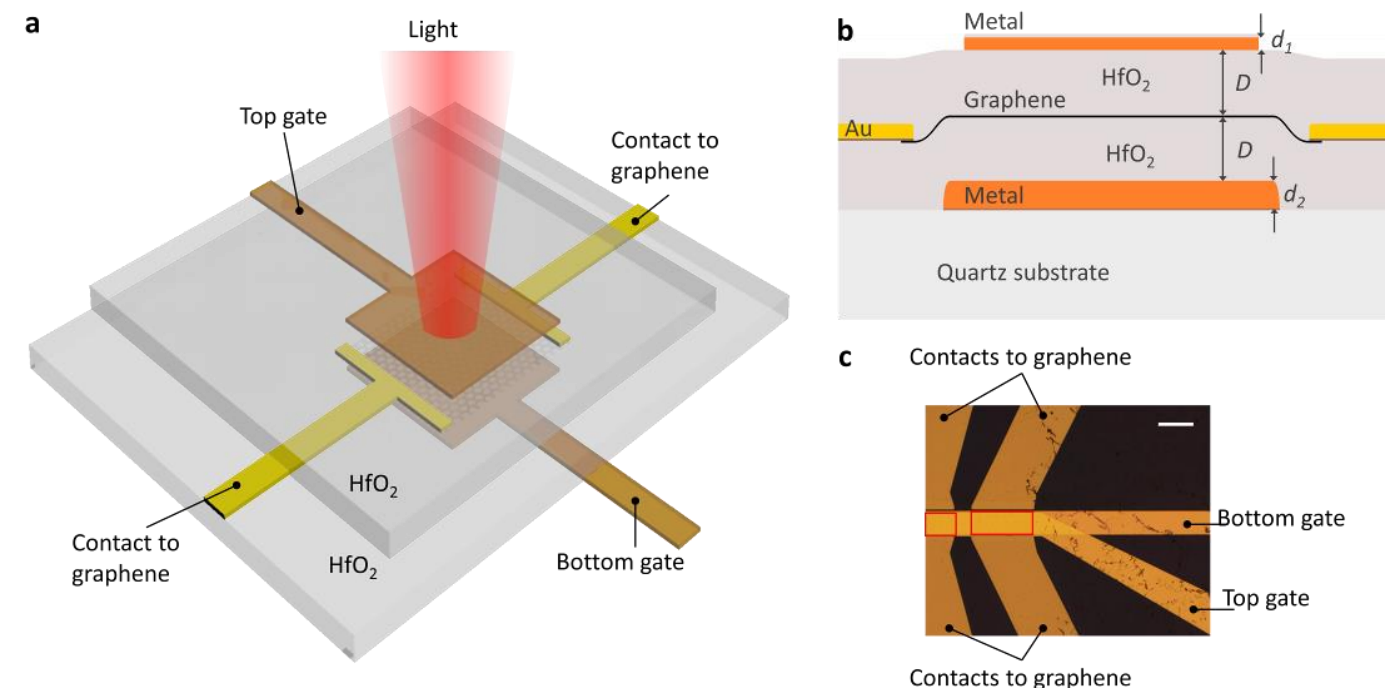

Figure 1. Optical modulator design. (a) Device schematic. (b) Cross-section of a device. (c) Optical microscope image of typical devices. The active areas of two devices are indicated by red rectangles. Scale bar $50 \mu \mathrm{m}$. 
a

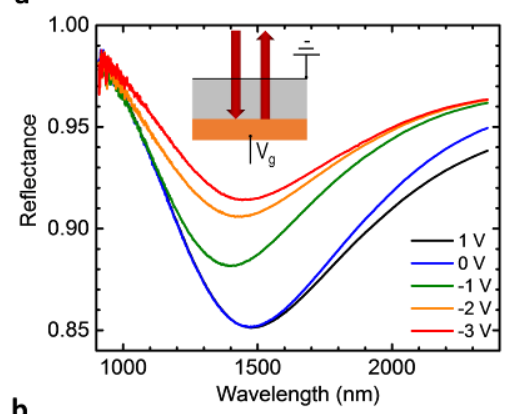

b

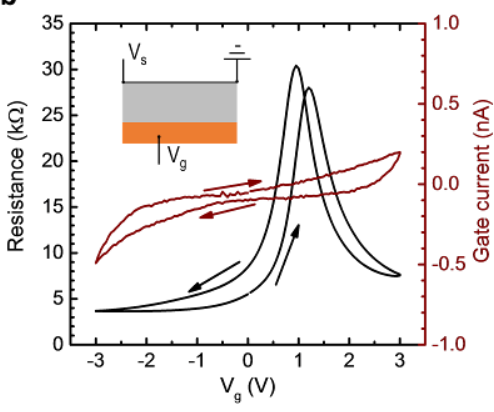

c

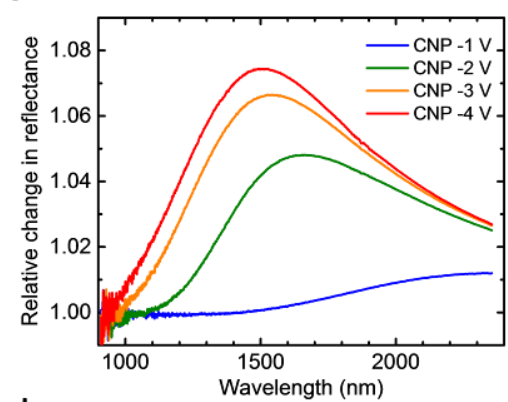

d

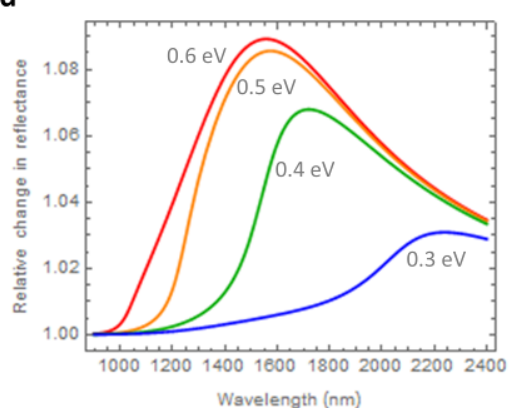

e

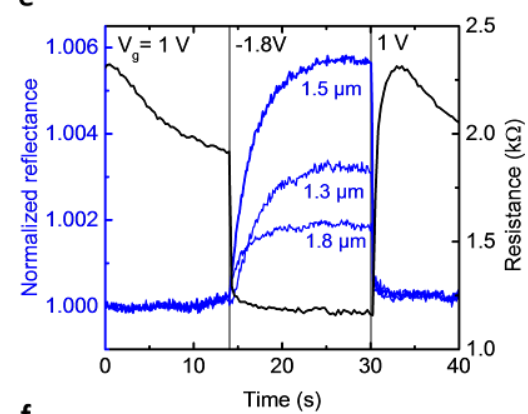

f

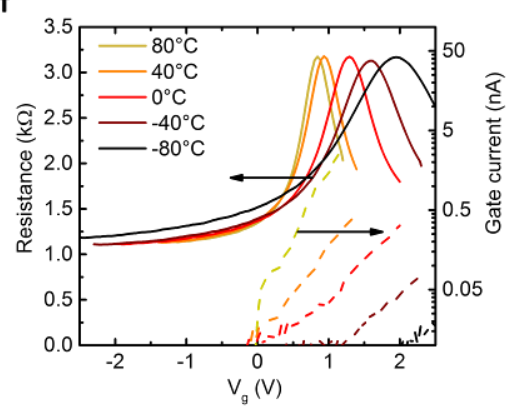

Figure 2. $\lambda / 4$ separator structure. (a) Optical reflectance spectra for different gate voltages. (b) Electrical resistance through graphene and gate current as a function of gate voltage (swept at $0.05 \mathrm{~V} / \mathrm{s}$ ). (c) Change in reflectance taking as reference the charge neutrality point (CNP) corresponding to gate voltage 1 V. (d) Numerical simulations for the relative change in reflectance of the device at different Fermi energies in graphene. (e) Time response of the graphene resistance and the device reflectance with gate voltage changes. (f) Temperature response of the graphene resistance and gate current as a function of the gate voltage.
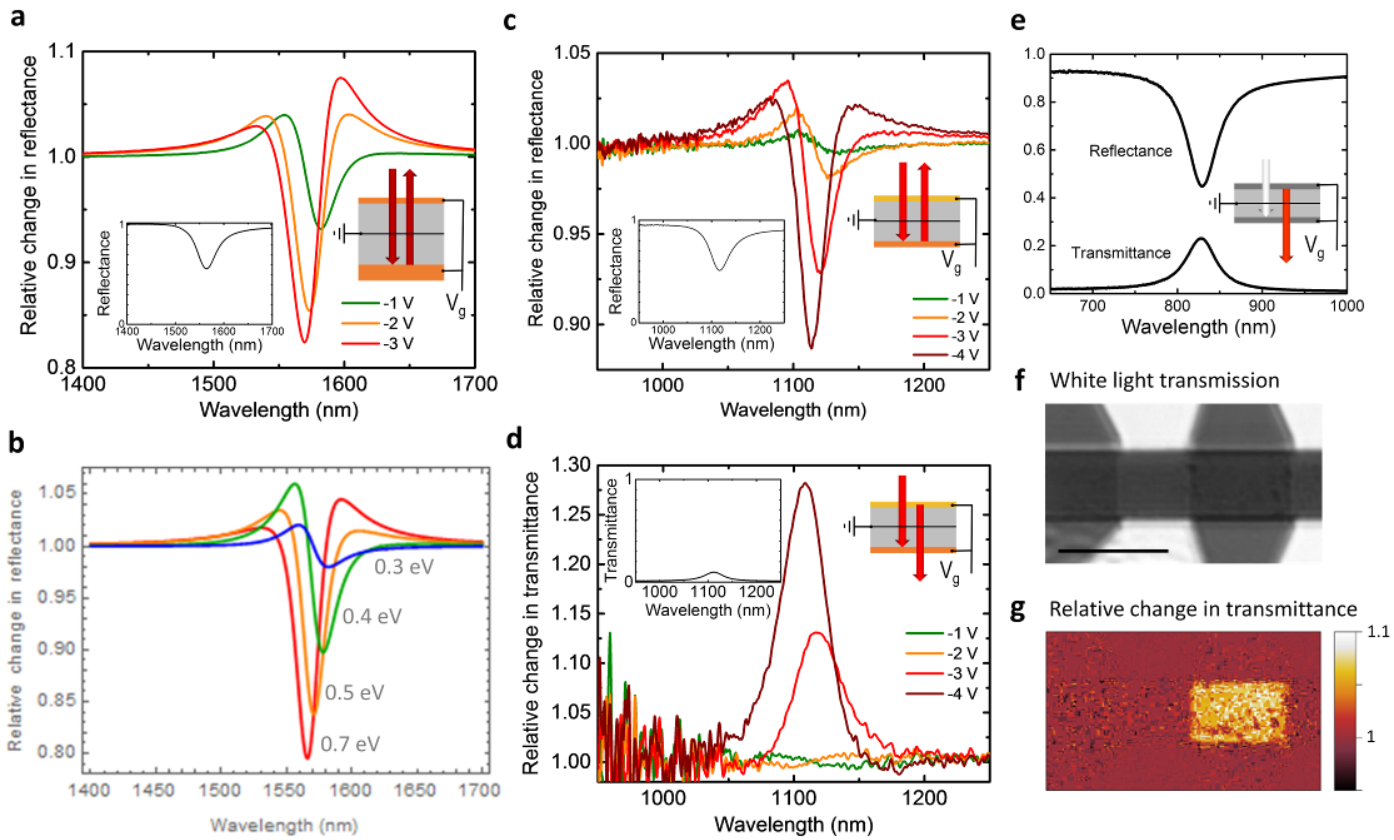
Figure 3. Fabry-Perot modulators. (a) Change in reflectance in a device designed for reflection at $1.5 \mu \mathrm{m}$ for different gate voltages relative to $0 \mathrm{~V}$ (shown in the inset). (b) Numerical simulations of the change in reflectance at different Fermi energies in graphene relative to $0 \mathrm{eV}$. (c and $\mathbf{d}$ ) Change in reflectance and transmittance in a device designed for transmission at $1.1 \mu \mathrm{m}$ for different gate voltages relative to $0 \mathrm{~V}$ (shown in the insets). (e) Reflectance and transmittance in a device designed for transmission at $830 \mathrm{~nm}$. (f) CCD camera image taken in transmission. Scale bar $50 \mu \mathrm{m}$. (g) Maximum change in transmittance when the top and bottom gates voltage is modulated with a $2 \mathrm{~Hz}$ sinusoidal signal from $-4 \mathrm{~V}$ to $4 \mathrm{~V}$.

a
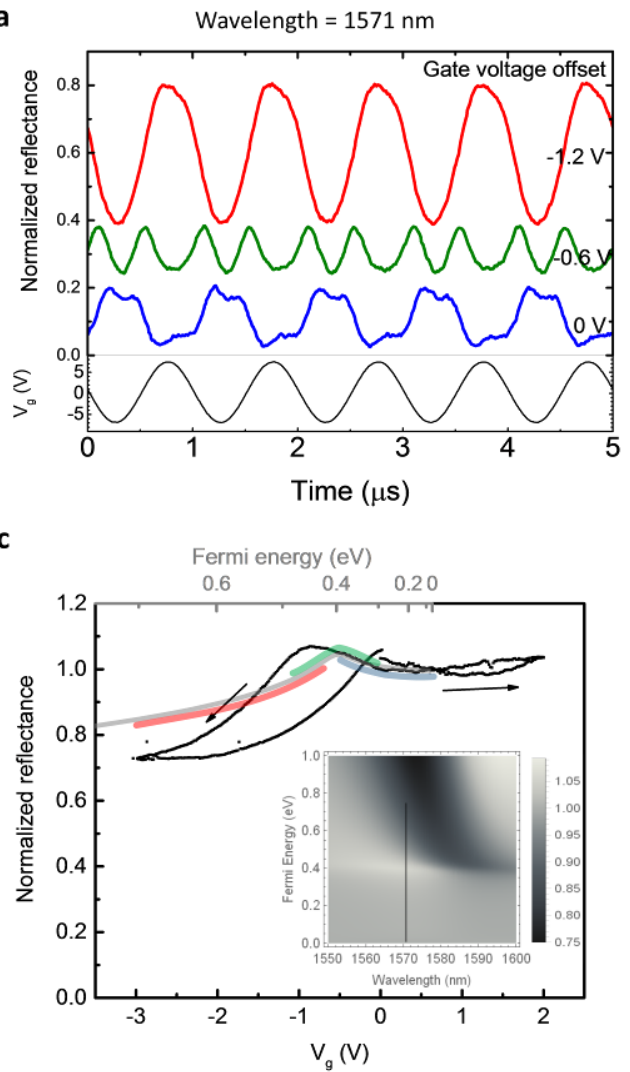

b

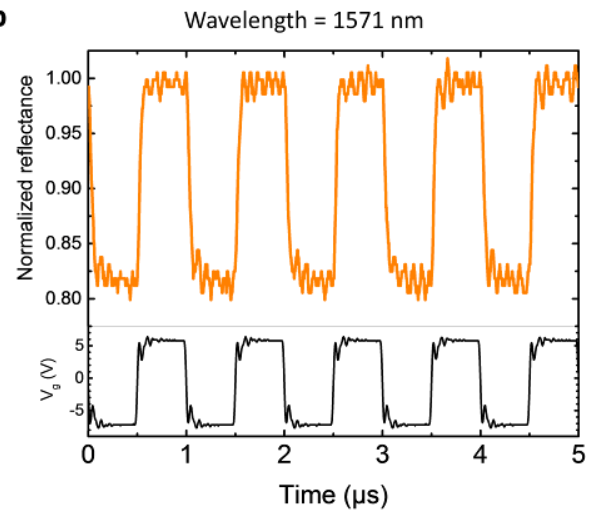

d

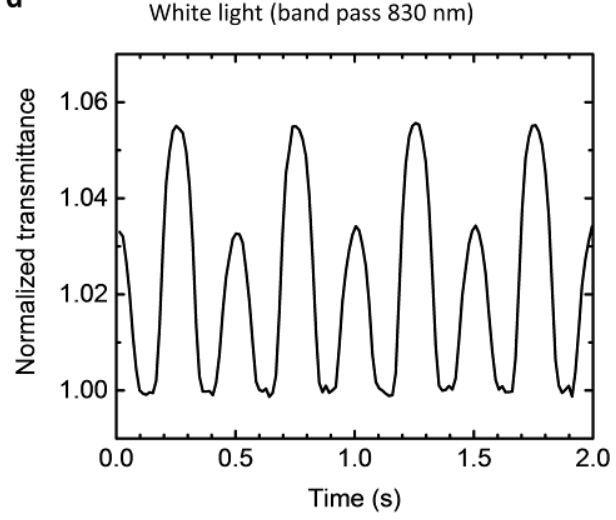

Figure 4. High frequency modulation. (a) Modulation of the reflectance at $1571 \mathrm{~nm}$ in the device from Figure $3 \mathrm{a}$. A $1 \mathrm{MHz}$ sine function of $16 \mathrm{~V}$ peak to peak amplitude with different voltage offsets is applied to the gate. The reflectance curves are normalized to the reflectance for zero gate voltage and shifted in the vertical axis for clarity. (b) Modulation with a square signal. (c) Black: Normalized reflectance as a function of the gate voltage (swept at 0.017 $\mathrm{V} / \mathrm{s}$ ). Gray: Numerical simulation of the reflectance as a function of the estimated Femi energy. Blue, green and red regions indicate the estimated regions of modulation of the similarly colored traces in (a). Inset: Numerical simulation of the change in reflectance as a function of the Fermi energy and the wavelength. (d) Modulation of the transmittance of white light (filtered by the $830 \mathrm{~nm}$ resonance) in the device from Figure 3c. A $2 \mathrm{~Hz}$ sine function of $8 \mathrm{~V}$ peak to peak amplitude is applied to top and bottom gates. 